\title{
Big Data in Food Industry
}

\author{
Matthew N. O. Sadiku1, Tolulope J. Ashaolu², Abayomi Ajayi-Majebi, ${ }^{3}$ and Sarhan M. Musa ${ }^{1}$
}

${ }_{1}^{1}$ Roy G. Perry College of Engineering, Prairie View A\&M University Prairie View, TX, USA

${ }^{2}$ College of Food Science, Southwest University, Tiansheng Road Beibei District, Chongqing, 400715, P.R. China

${ }^{3}$ Department of Manufacturing Engineering, Central State University, P.O. Box 1004 Wilberforce, OH 45384-1004, USA

E-mail: sadiku@ieee.org; ashaolut@gmail.com; ajayi-majebi@centralstate.edu; smmusa @pvamu.edu

*Corresponding author details: Assoc. Prof. Matthew N.O. Sadiku, sadiku@ieee.org

\begin{abstract}
Big data is changing the world around us. It is changing the food industry for the better. The food industry has huge potential for applying big data solutions as the major food industry players can use data analytics to empower their business. Many food companies are leveraging data analytics to design their inventory, boost business, reduce expenses, improve quality control, meet changing demands, improve consumer experience, reduce waste, and save resources. This paper provides several opportunities for big data applications in food industry.
\end{abstract}

Keywords: food industry; big data; data analytics

\section{INTRODUCTION}

Food is one of the necessities of life. We cannot survive without food. A popular Chinese proverb says, "the masses regard food as their prime want." We are programmed to want food that is tasty, fresh, and healthy. This makes food to be highly in demand and relevant in today's world. The food industry is the most indispensable and most substantial sector in the world. It encompasses farming, food producers, food manufacturers, packers, cooks, food and beverage companies, shipping companies, to supermarkets, and restaurants [1].

Data is becoming part of our lives. Big data refers to the information which cannot be processed with the help of traditional tools. It is a huge volume of data which is being generated worldwide in nearly all sectors of the society. As more of this big data becomes available, it can be used to gain new insights, improve decisionmaking, and enhance the quality of products and services [2]. Big data and analytics are helping to improve and transform a multitude of industries in the modern world, and food industry is no exception. Big data has been around for many years but the food industry has just started to use it to its full potential. The food industry across the world is facing an intere sting challenge. It generates large amounts of data every day.

\section{BIG DATA CHARACTERISTICS}

Big data (BD) is a relatively newer technology that can help the food industry. The three main sources of big data are machines, people, and companies. As shown in Figure 1 [3], big data can be described with 42 Vs. The first five Vs are volume, velocity, variety, veracity, and value [4].

- Volume: This refers to the size of the data being generated both inside and outside organizations and is increasing annually. Some regard big data as data over one petabyte in volume.
- Velocity: This depicts the unprecedented speed at which data are generated by Internet users, mobile users, social media, etc. Data are generated and processed in a fast way to extract useful, relevant information. Big data could be analyzed in real time, and it has movement and velocity.

- Variety: This refers to the data types since big data may originate from heterogeneous sources and is in different formats (e.g., videos, images, audio, text, logs). BD comprises of structured, semi-structured or unstructured data.

- Veracity: By this, we mean the truthfulness of data, i.e. weather the data comes from a reputable, trustworthy, authentic, and accountable source. It suggests the inconsistency in the quality of different sources of big data. The data may not be $100 \%$ correct.

- Value: This is the most important aspect of the big data. It is the desired outcome of big data processing. It refers to the process of discovering hidden values from large datasets. It denotes the value derived from the analysis of the existing data. If one cannot extract some business value from the data, there is no use managing and storing it.

On this basis, small data can be regarded as having low volume, low velocity, low variety, low veracity, and low value. Additional five Vs has been added [5]:

- Validity: This refers to the accuracy and correctness of data. It also indicates how up to date it is.

- Viability: This identifies the relevancy of data for each use case. Relevancy of data is required to maintain the desired and accurate outcome through analytical and predictive measures. 
- Volatility: Since data are generated and change at a rapid rate, volatility determines how quickly data change.

- Vulnerability: The vulnerability of data is essential because privacy and security are of utmost importance for personal data.

- Visualization: Data needs to be presented unambiguously and attractively to the user. Proper visualization of large and complex clinical reports helps in finding valuable insights.

Instead of the 5V's above, some suggest the following $5 V$ 's: Venue, Variability, Vocabulary, Vagueness, and Validity) [6].

To thrive in today's complex business environment, businesses must adopt a data-driven culture and leverage analytics platforms to make key decisions that improve productivity. Industries that benefit from big data include the healthcare, financial, airline, travel, restaurants, automobile, sports, agriculture, and hospitality industries. Big data technologies are playing an essential role in farming: machines are equipped with sensors that measure data in their environment

\section{BIG DATA ANALYTICS}

Every day, data is growing bigger and bigger, and big data analysis (BDA) has become a requirement for gaining invaluable insights into data such that companies could gain significant profits in the global market. Once the big data is ready for analysis, we use advanced software programs such as Hadoop, MapReduce, MongoDB, and NoSQL databases [7]. Big data analytics refers to how we can extract, validate, translate, and utilize big data as a new currency of information transactions. It is an emerging field that is aimed at creating empirical predictions. Data-driven organizations use analytics to guide decisions at all levels [8].

Data scientists know how to use tools that identify patterns and relationships that may otherwise remain hidden. They are part of virtually every major industry, and agriculture is no exception. Agricultural big data analytics is expected to ensure better farming practices, decision-making, and a sustainable future for humankind. This will involve artificial intelligence and machinelearning technologies to determine better farming practices and decision-making [9].

One-way food industry can take more control of their quality parameters is through data analytics. To stay competitive and keep pace with consumers' fickle buying habits, the food industry must consider implementing data analytics tools. The good thing about data analytics is that it can be implemented anywhere in the world.

\section{WHY BIG DATA IN FOOD INDUSTRY?}

Data were always regarded as an important source of knowledge for farmers, agricultural professionals, and policy-makers. Farming has been empirically driven for over a century but the data collected was not digital. The agriculture community deals with a massive amount of structured and unstructured data. Use of big data is revolutionizing the food industry in many ways due to the large amount of information and insights it offers. Here are some typical examples of how big data is revolutionizing the food industry.
- Fast Delivery: Timing is everything in the food industry. Getting the food product delivered to the customer in a timely manner is a priority. To facilitate rapid deliveries big data analytics can be employed for monitoring traffic, construction weather, changes in routes, present climates, route changes, construction, and distance [10]. Big data can optimize on-time deliveries of orders to restaurants and customers at home. For example, when pizza arrives at your home in less than 30 minutes, the big data systems calculates delivery time, distance, and even traffic levels. By using predictive analytics, a food delivery app can estimate precisely how many customers will order during a particular time of the day. Figure 2 shows how big data analytics helps food delivery [11].

- Quality Control: In the food industry, quality is a very important factor, but keeping quality consistent in a competitive market is an uphill task. Quality is one of the factors that dictate food's taste. Big data is vitally important for food quality control. It plays a crucial role in the overall quality of food. By using big data, it is possible to replace any damaged products with proper ones on time [10].

- Improved Efficiency: In an industry that affects the majority of population, any improvement in efficiency always has a far-reaching effect. For example, restaurant chains are exploring how big data can help them improve their business. The fast food chain uses big data analytics to optimize the drive-thru experience. Looking for trends in increased consumer demand can be particularly beneficial for improving efficiency and preparing for that spike in demand ahead of time [12]

- Sentiment Analysis: Sentiment is the index that measures what is said about a brand or a product. Sentiment analysis is a technique that is used by many businesses to learn more about their customers and growing sentiment about a brand. Food businesses employ sentiment analysis to track their customers' emotions. Big data helps in assessing customer emotions that are expressed on social media networks like Twitter or Facebook. Sentiment analysis is used in the food and beverage industry to understand trends and popular items or goods. Big data is making sentiment analysis much easier [13]

- Personalization: The ultimate aim of several big data projects is to provide a personalized, customercentric experience to every consumer. This involves analyzing customer views such as what they like, how much they are willing to pay, the reviews and stories they share on social media, etc. Big data analytics has made possible the tracking, collection, and analysis all this data [14].

Other ways big data can improve the food industry include product pricing, demand forecasting, loyalty, payments, consumer service, supply chain transparency, mobile apps and modern experiences, market basket analysis, and predicting snack food trends.

\section{APPLICATIONS OF BIG DATA IN FOOD INDUSTRY}

The applications of big data in the food industry are so diverse and extensive that from production to customer service everything can be optimized. Every stakeholder, starting with farmers and ending with restaurants, is required to have relevant data on their products. Here are a few examples of the real-world applications of big data in the food industry 
- Agriculture: Today, data is transforming one of the world's oldest industry: agriculture. Agriculture has become increasingly high-tech and data-driven over the years. Tough issues such as a growing world population and changing dietary habits are affecting agricultural production. Big data is inevitable in agriculture. Its potential to "revolutionize" agricultural industry is of interest to the industry and researchers across the world. The sector is just starting to implement big data and is already has impressive results [15].

- Farming: The farming sector constitutes some sort of basement for all food industries. It is undergoing a digital revolution. Big data has a huge potential to lift farmers out of poverty. Big data analytics enable farmers to detect problems early on and take necessary action. This improves production and efficiency of the farm. Big data can help farming to dramatically increase the crop yield. A lot of data is being generated in the farming industry: data from sensors, irrigation data, weather condition, the seed yield, crop maturity time, etc. These data can be analyzed to provide unimaginable insight to farmers, which would help them make right decisions [16]. Applications of big data in smart farming will raise many power-related issues. Big data is changing the organization of farming through a pull-push mechanism [17].

- Restaurant: Due to a huge number of competitors, the restaurant business is risky. Big data provides great opportunities for restaurant owners, allowing them to compare gathered data and choose the most efficient strategy. Restaurant data analytics involves gathering data and analyzing it to obtain crucial insights. Restaurant analytics software can monitor the market condition and adjust to new trends. For example, KFC uses big data to analyze customer feedback and food preferences [10].

- Transportation: In a globalizing world, the transportation of produce and products across countries and continents has been normalized. Data analytics and data prediction technologies offer powerful solutions for food transporters. Big data analytics can provide valuable analytics insights on transportation of the farm produce. Big data also helps in determining the best transportation method and helps in optimizing routes [18]

- Food Security: Global hunger is one of the pressing and moral challenges facing humanity today. We face the global challenge to feed a world of 10 billion people by 2050. Improving food security faces a number of data challenges. Big data analytics has the potential to transform key operations in the food and beverage industry and address food security challenges around the globe. This is achieved by reforming the process of how data is collected, combined, analyzed, and shared [19]. Attaining food security is ensuring food availability, which depends on food production.

- Food Safety: Food safety has taken the center stage, as the world is more connected than ever before. With dense populations of people around the world, the production and delivery of food has raised serious food safety concerns. Big data analytics can address this concern by helping food industry players to analyze data regarding food safety. Big data offers both preventative and reactive solutions to food safety. Analyzing data can also help in faster recalls and driving automation thus, saving a significant amount of money [20].

- Food Waste: Restaurants and supermarkets are significant contributors to food waste in landfills that release harmful methane gas into the environment. Some of the factors responsible for food waste include consumers' demand for fresh products, damaged delivery of products, and over-purchasing. Sustainable alternatives are becoming a mandatory requirement. Data is crucial to understanding what is being produced and what is being thrown away. Big data provides the information that allows us to better address the problem of food waste [21].

\section{BENEFITS AND CHALLENGES}

Big data is increasingly present in our daily lives without even realizing it most of the time. Big data on the food industry has several benefits. Big data can significantly change the entire food industry and streamline profit. It assists food businesses in improving their marketing campaigns and developing high demand products. It can to help businesses create new products or redesign existing brands. It provides huge opportunities for creation of new businesses, development of new products, and improvements in existing business operations. Big data helps food businesses enhance product innovation, improve their marketing campaigns, improve sales effectiveness, boost profitability, and improve customer satisfaction. It also plays a big role in supply chain transparency.

Adaption of any disruptive technology, such as big data, can be daunting. Food businesses still face challenges in exploiting big data and making investment decisions. This is due to data processing speed, interpretation, quality, security and privacy, and shortage of qualified data scientists. Data collected from social media and other Internet-based sources may be subjected to consumer sentiments. The data becomes unreliable and uncertain due to subjectivity of human opinions. Big data and its tools are provoking a host of ethical concerns [22]. A big challenge of big data governance is ensuring privacy and security.

\section{CONCLUSION}

The food industry is one of the most profitable sectors globally. Big data is critical in helping the food industry meet the challenges of growing world population, climate change, and urbanization. The applications of big data in the food industry are expanding rapidly. Big data is improving and streamlining experiences that companies like McDonald's, KFC, Taco Bell, Subway, Burger King, and others. Big data is poised to revolutionize the food industry and reproduce long-standing relationships between food system players.

\section{REFERENCES}

[1] Namaste UI, "Astounding possibilities of big data in the food industry," July 2019, https://www.namasteui.com/astoundingpossibilities-of-big-data-in-the-food-industry/

[2] J. P. Marvin et al., "Big data in food safety: An overview," Critical Reviews in Food

Science and Nutrition, vol. 57, no. 11, 2017, pp. 2286-2295.

[3] "The $42 \mathrm{~V}$ 's of big data and data science," https://www.kdnuggets.com/2017/04/42-vs-bigdata-data-science.html 
[4] M. N.O. Sadiku, M. Tembely, and S.M. Musa, "Big data: An introduction for engineers," Journal of Scientific and Engineering Research, vol. 3, no. 2, 2016, pp. 106-108.

[5] P. K. D. Pramanik, S. Pal, and M. Mukhopadhyay, "Healthcare big data: A comprehensive overview," in N. Bouchemal (ed.), Intelligent Systems for Healthcare Management and Delivery. IGI Global, chapter 4, 2019, pp. 72-100.

[6] J. Moorthy et al., "Big data: Prospects and challenges," The Journal for Decision Makers, vol. 40, no. 1, 2015, pp. 74-96.

https://www.grandviewresearch.com/industryanalysis/industrial-wireless-sensor-networksiwsn-market

[7] M. N. O. Sadiku, J. Foreman, and S. M. Musa, "Big data analytics: A primer," International Journal of Technologies and Management Research, vol. 5, no. 9, September 2018, pp. 44-49.

[8] C. M. M. Kotteti, M. N. O. Sadiku, and S. M. Musa, "Big data analytics," Invention Journal of Research Technology in Engineering \& Management, vol. 2, no. 10 , Oct. 2018 , pp. $2455-3689$.

[9] M. Ryan, "Agricultural big data analytics and the ethics of power," Journal of Agricultural and Environmental Ethics, vol. 33, 2020, pp. 49-69.

[10] M. Rangaiah, "Role of big data in the food industry," April 2020,

Https://www.analyticssteps.com/blogs/role-bigdata-food-industry

[11] "Big data is 'delivering' results for food delivery apps,"https://prismetric.weebly.com/blog/bigdata-analytics-for-food-delivery-apps

[12] "How big data is revolutionizing the food industry,"

https://www.wired.com/insights/2014/02/bigdata-revolutionizing-food-industry/

[13] N. Sykes, "5 uses of big data in the food industry," July 2018, https://www.kolabtree.com/blog/5uses-of-big-data-in-the-food-industry/

[14] Anurag, "7 Uses of big data in food and beverages industry," September 2017,

https://www.newgenapps.com/blog/7-uses-ofbig-data-in-food-and-beverages-industry/

[15] M. N. O. Sadiku, T. J. Ashaolu, and S. M. Musa, "Big data in agriculture," International Journal of Scientific Advances, vol. 1, no.1, July-August 2020.

[16] Arcgate, "Food industry and technology," January 2019,https://medium.com/@arcgate/foodindustry-and-technology-a7af4456b86a

[17] S. Wolfertab, L. aCorVerdouwab, and M. J. Bogaardta, "Big data in smart farming - A review," Agricultural Systems, vol. 153, May 2017, pp. 69-80.

[18] "Analyzing the role of big data in transforming the food industry | Quantzig," https://www.businesswire.com/news/home/202 00624005295/en/Analyzing-the-Role-of-Big-Datain-Transforming-the-Food-Industry-Quantzig

[19] "How is big data transforming the food industry? | Head to Quantzig's recent article to find out," July 2020,https://www.businesswire.com/news/home /20200715005325/en/How-Is-Big-Data-

Transforming-the-Food-Industry-Head-toQuantzig\%E2\%80\%99s-Recent-Article-to-Find-out
[20] "How big data plays a role in food safety," https://sprimfood.com/how-big-data-plays-arole-in-food-safety-detecting-alerts-monitoringenvironments-and-anticipatingneeds/\#: :text=Insights\%20from $\% 20 \mathrm{big} \% 20$ dat a\%20are,our\%20globalizing\%20and\%20intercon nected $\% 20$ world.\&text=Big\%20Data\%20is\%20s pecifically $\% 20$ being,free $\% 20$ from $\% 20$ food $\% 20$ sa fety\%20hazards.

[21] "Grocers can use big data, analytics to reduce food waste,"https://www.stcr.com/blog/grocers-canuse-big-data-analytics-reduce-food-waste/

[22] N. Dunford, "Big data and opportunities for agricultural and food industries," December 2017, https://extension.okstate.edu/fact-sheets/bigdata-and-opportunities-for-agricultural-and-foodindustries.html

\section{ABOUT AUTHORS}

Matthew N.O. Sadiku is a professor emeritus in the Department of Electrical and Computer Engineering at Prairie View A\&M University, Prairie View, Texas. He is the author of several books and papers. His areas of research interests include computational electromagnetics and computer networks. He is a fellow of IEEE.

Tolulope J. Ashaolu works at Southwest University, P.R.C. He is the author of several papers and a book. His research interests include functional foods and food microbiology.

Abayomi Ajayi-Majebi is a professor in the Department of Manufacturing Engineering at Central State University in Wilberforce, Ohio. In 2015 he was honored by the White House as a Champion of Change for his significant contributions to the engineering education of minority students. He is a senior member of both the Society of Manufacturing Engineers and the American Society for Quality.

Sarhan M. Musa is a professor in the Department of Electrical and Computer Engineering at Prairie View A\&M University, Prairie View, Texas. He has been the director of Prairie View Networking Academy, Texas, since 2004. He is an LTD Sprint and Boeing Welliver Fellow. His areas of research interests include computational electromagnetics and computer networks. 


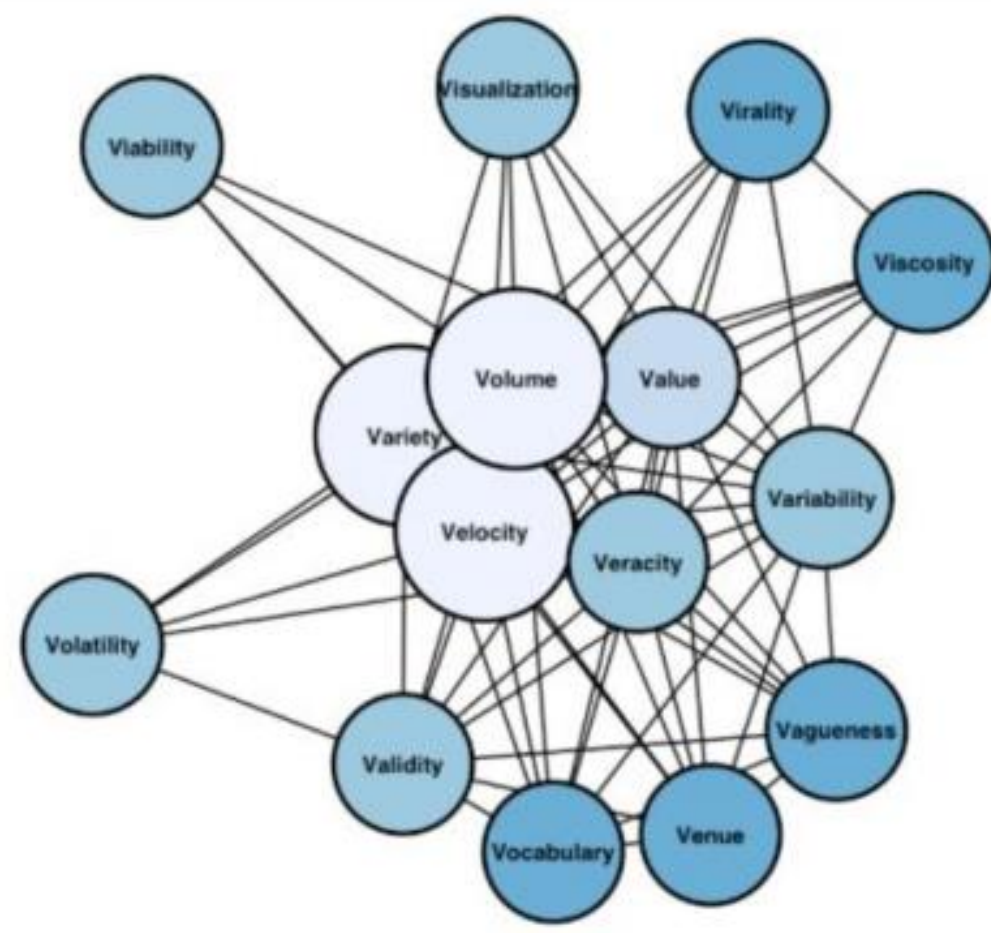

First Occurrence

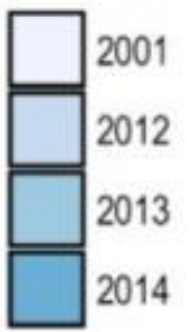

FIGURE 1: THE 42 V' S OF BIG DATA [3].
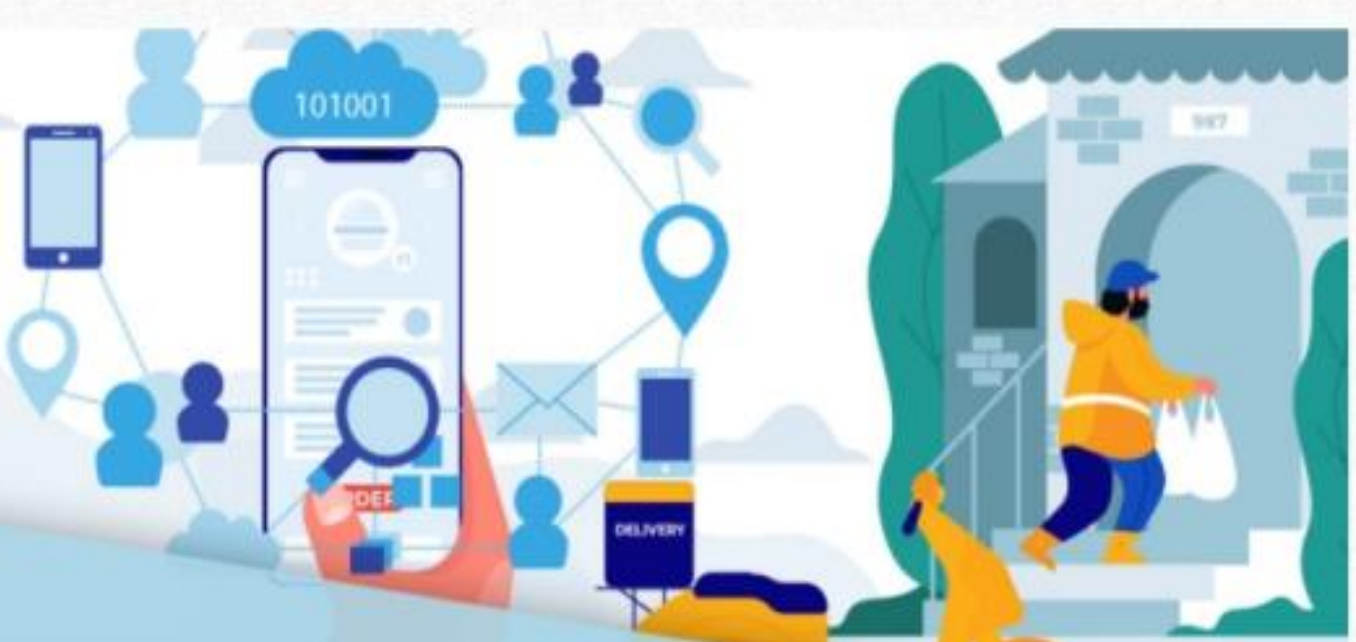

How Big Data Analytics helps the Food Delivery Apps?

FIGURE 2: BIG DATA ANALYTICS HELPS FOOD DELIVERY [11]. 